\title{
El cuidado crítico en el paciente con COVID-19
}

\section{Critical care in the patient with COVID-19}

\section{Criterios de ingreso a la Unidad de Cuidado Intensivo (UCI)}

A comienzos de la pandemia se planteó que la mortalidad se relacionaba de forma inversa con la disponibilidad de recursos sanitarios. Debido a esto se buscó expandir las camas de cuidado intensivo y el número de ventiladores disponibles antes del pico. Si bien tal conducta ha sido cuestionada, está claro que resulta fundamental contar con una herramienta que permita seleccionar a los pacientes que requieren manejo en UCI, con base en una predicción de morbimortalidad.

Por tratarse de una neumonía, en la gran mayoría de los casos, los lineamientos del Ministerio de Salud y el consenso de la Asociación Colombiana de Infectología (ACIN) propusieron los criterios de la American Thoracic Society (ATS) del 2007. Así, la presencia de un criterio mayor o tres criterios menores justificarían el ingreso a UCI (Tabla 1):

- Criterios mayores: necesidad de ventilación mecánica, presencia de choque séptico

- Criterios menores: frecuencia respiratoria $\geq 30 \mathrm{rpm}$; presión arterial de oxígeno/fracción inspirada de oxígeno $\left(\mathrm{PaO}_{2} / \mathrm{FiO}_{2}\right)<250$; compromiso multilobar; confusión/desorientación; BUN $\geq 20 \mathrm{mg} / \mathrm{dL}$; leucopenia $<4000 / \mathrm{mm}^{3}$; presión arterial sistólica (PAS) $<90 \mathrm{~mm} \mathrm{Hg}$; hipotensión que requiere aporte intensivo de líquidos; temperatura central $<36{ }^{\circ} \mathrm{C}$; plaquetas $<100000 / \mathrm{mm}^{3}$.

Por otro lado, la Asociación Colombiana de Medicina Crítica y Cuidado Intensivo (AMCI) propuso la escala NEWS como herramienta práctica, basada en parámetros clínicos fáciles de evaluar. Una sumatoria de puntos mayor o igual de 7 sería el criterio para ingreso a UCI (1). Está claro que la saturación arterial de oxígeno $\left(\mathrm{SaO}_{2}\right)$ requiere un ajuste, dependiendo de la altura sobre el nivel del mar en que se encuentre (2).
${ }^{1}$ Médico especialista en Medicina Interna y Neumología, miembro del Comité de Cuidado Crítico de la Asociación Colombiana de Neumología. Autor de correspondencia: Carmelo Dueñas-Castell, MD. Correo electrónico: crdc2001@gmail.com Recibido: 20/06/2020 Aceptado: 01/07/2020 
Tabla 1. Criterios de ingreso a la unidad de cuidado intensivo

\begin{tabular}{|lccccccc|}
\hline \multicolumn{1}{|c}{ Parámetros } & $\mathbf{3}$ & $\mathbf{2}$ & $\mathbf{1}$ & $\mathbf{0}$ & $\mathbf{1}$ & $\mathbf{2}$ & $\mathbf{3}$ \\
\hline Edad & & & & $<65$ & & $\geq 65$ \\
\hline $\begin{array}{l}\text { Frecuencia respiratoria } \\
\text { Saturación de oxígeno }\end{array}$ & $<8$ & & $9-11$ & & & $>25$ \\
$\begin{array}{l}\text { Cualquier suplemento de } \\
\text { oxígeno }\end{array}$ & & $92-93$ & $94-95$ & $>96$ & & \\
\hline $\begin{array}{l}\text { Presión arterial sistólica } \\
\text { Frecuencia cardíaca }\end{array}$ & $<90$ & $91-100$ & $101-100$ & $111-219$ & & & $>220$ \\
\hline Temperatura & $<40$ & & $41-50$ & $51-90$ & $91-110$ & $111-130$ & $>131$ \\
\hline
\end{tabular}

\section{Oxigenoterapia}

Las recomendaciones para suministrar oxígeno al paciente crítico con COVID-19 han surgido de guías publicadas antes y durante la pandemia. Estas se pueden resumir en las siguientes recomendaciones generales $(1,3,4-6)$ :

- Suministrar oxígeno a pacientes con COVID-19 para lograr metas mayores de $94 \%$.

- Iniciar oxígeno a 4-5 L/minuto, y valorar el flujo para mantener la $\mathrm{SaO}_{2}>93 \%$ durante la reanimación, o usar máscara facial con bolsa de reservorio (10-15 L/min), si el paciente está en condición crítica.

- Una vez el paciente se encuentre estable, la meta de $\mathrm{SaO}_{2}$ es $>90 \%$ en adultos, y $>92 \%-95 \%$ en embarazadas.

\section{Medicamentos}

Desde el principio de la pandemia se reportó que la fisiopatología del COVID-19 se caracterizaba por etapas que esquemáticamente se podrían resumir en una inicial, con una alta carga viral, seguida por un segundo período de hiperinflamación, que podría generar una tormenta de citocinas (Figura 1).

Con base en ello se plantearon medicamentos antivirales para la infección temprana e inmunosupresores para los estadios más avanzados $(7,8)$. Tales intervenciones se usaron sin tener mayor evidencia, con el único soporte de la plausibilidad biológica, y con base en estudios clínicos controlados, como el RECOVERY y el SOLIDARITY, en los que se demostraron que no generaban beneficio alguno comparado con el cuidado estándar en la UCI, y que, además, podrían generar eventos adversos (7-10). Luego de varios meses de pandemia, al enfrentar a pacientes críticos solo existe evidencia para el uso rutinario de dexametasona $6 \mathrm{mg} /$ día IV en pacientes hipoxémicos o en ventilación mecánica por 10 días, o hasta que se logre el egreso hospitalario (11).

En junio de 2020 se publicaron los resultados preliminares de uno de los grupos del ensayo clínico del estudio RECOVERY (11). En este estudio se compararon 2104 pacientes asignados al azar para recibir dexametasona frente a 4321 pacientes asignados simultáneamente al tratamiento habitual. Los pacientes se asignaron en una proporción de 2:1 al tratamiento estándar o habitual o al tratamiento habitual más $6 \mathrm{mg}$ de dexametasona 1 vez al día (oral o intravenosa), durante un máximo de 10 días (o hasta el alta, si es antes). $454(21,6 \%)$ pacientes asignados a la intervención con dexametasona y 1065 (24,6\%) pacientes asignados al tratamiento habitual fallecieron a los 28 días (índice de frecuencia ajustada por edad [RR 0,83; IC 95\% 0,74 a $0,92 ; P<0,001)]$.

Las reducciones de la tasa de mortalidad proporcional y absoluta variaron significativamente, dependiendo del nivel de soporte respiratorio en el momento de la aleatorización $(p<0,001)$. El uso de dexametasona redujo la mortalidad a los 28 días un $35 \%$ en pacientes que recibieron ventilación mecánica invasiva (RR 


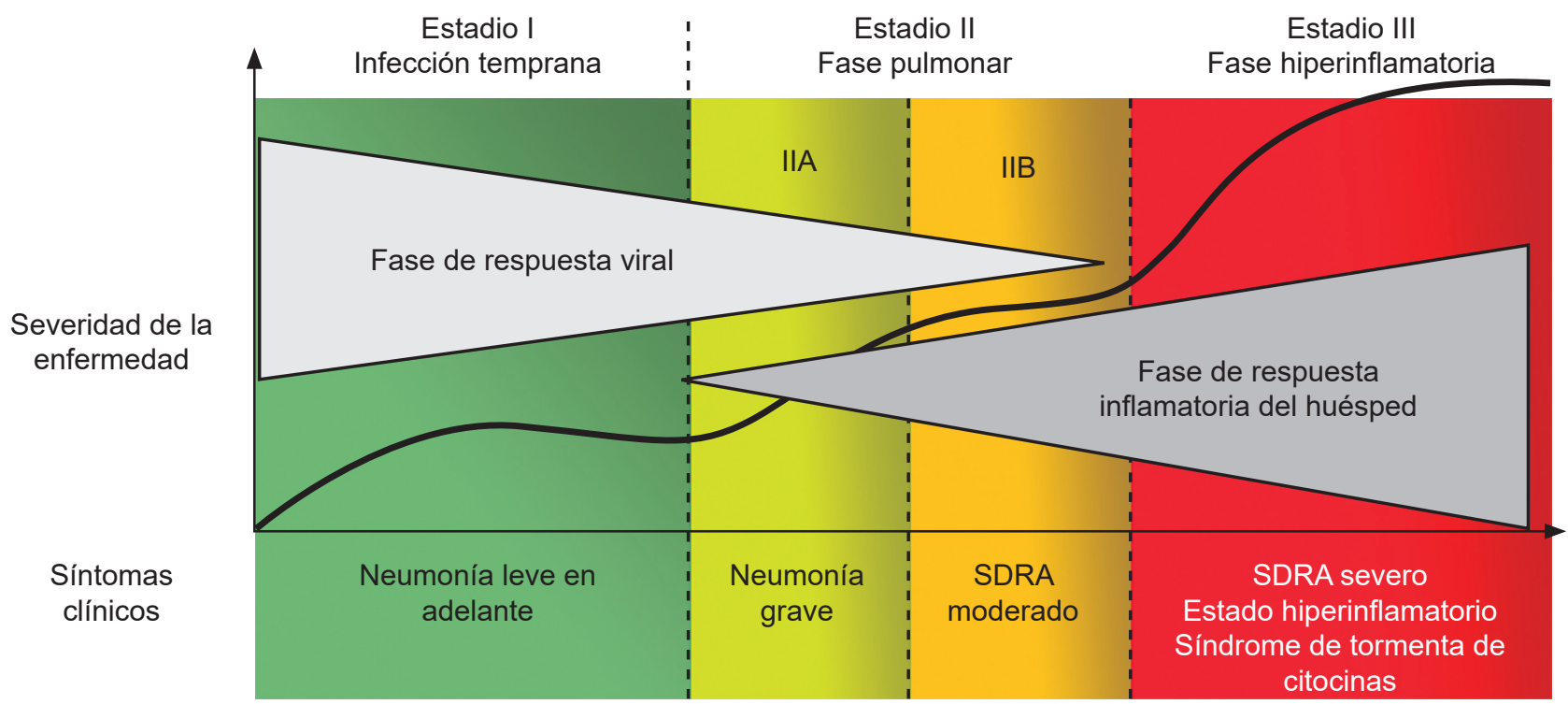

Figura 1. Resumen esquemático de las etapas, que va desde una etapa inicial, con una alta carga viral, seguida por un segundo período de hiperinflamación.

SDRA: síndrome de dificultad respiratoria aguda.

$0,65$; IC $95 \% 0,51$ a 0,$82 ; p<0,001)$ y $20 \%$ en pacientes que recibieron oxígeno sin ventilación mecánica invasiva (RR 0,80; IC 95\% 0,70-0,92; $p=0,002$ ). Sin embargo, no hubo evidencia de beneficio entre aquellos pacientes que no estaban recibiendo soporte respiratorio (RR 1,22; IC 95\% 0,93-1,61; $p=0,14$ ). La evaluación de grupos específicos define un beneficio de la intervención con dexametasona en pacientes menores de 70 años (RR 0,64; IC 95\% 0,52-0,78) con más de 7 días de síntomas (RR 0,68; IC 95\% 0,58-0,80).

En este grupo de pacientes se ha planteado la discusión sobre la alta frecuencia de hipercoagulabilidad y enfermedad tromboembólica; de allí derivan propuestas de anticoagulación. Esto no ha sido confirmado, y la mayoría de guías recomiendan iniciar la profilaxis tromboembólica en todo paciente crítico, a menos que haya contraindicación $(1,3,12,13)$. Hasta el momento no hay acuerdo sobre el uso de dosis más altas o anticoagulación plena, esto debería estar sujeto a una evaluación individual que considere el riesgo/beneficio.

\section{Medidas no invasivas}

Dada la severidad de la hipoxemia, que predomina en los pacientes con COVID-19 severo, se han busca- do diversas estrategias. Hace más de 50 años se planteó que la posición prona (PP) mejoraba la oxigenación en pacientes con falla respiratoria hipoxémica (14).

La mejoría en la oxigenación que produce la PP es multifactorial, ya que genera cambios en la distribución de la ventilación alveolar; mejora el tamaño alveolar; optimiza la relación ventilación/perfusión; puede reducir la lesión pulmonar inducida por el ventilador (especialmente barotrauma y atelectrauma); y mejora el desempeño del ventrículo derecho al aumentar el retorno venoso y reducir la poscarga y la resistencia vascular pulmonar (15).

Antes de la pandemia ya se había planteado el beneficio de la PP en pacientes no intubados al mejorar la oxigenación, y probablemente evitar la intubación (16, 17). Con el comienzo de la pandemia por COVID-19, y ante las limitaciones en disponibilidad de ventiladores y la aparente alta mortalidad en ventilación mecánica invasiva, se presentaron experiencias de PP en pacientes conscientes no intubados $(1,18,19)$.

Al comienzo de la pandemia, y ante los riesgos de contagio por generación de aerosoles, varias guías generaron alertas para evitar el uso de la cánula de alto 
flujo (CAF) y la ventilación mecánica no invasiva (VMNI) $(5,6)$. Está claro que ambas medidas producen dispersión de aerosoles, por tanto, lo ideal es realizarlo en salas de presión negativa $(1,3,5,6,13)$; sin embargo, dado que se ha demostrado que la dispersión de aerosoles es limitada, con el uso de elementos de protección personal (EPP) adecuados los riesgos se reducen notoriamente $(20,21)$. Más aún cuando se le coloca una mascarilla quirúrgica o de uso médico con CAF al paciente, y se usa doble filtro en los circuitos del ventilador en VMNI $(1,3,5,6,13)$.

La experiencia de PP en pacientes no intubados en el escenario de la pandemia ha crecido; de ella se puede concluir que, en general, es bien tolerada; es una intervención simple y de bajo costo; genera beneficios en la oxigenación; puede retardar el deterioro respiratorio en pacientes seleccionados; puede emplearse unida con estrategias de oxigenación no invasiva, como la CAF y la VMNI. Ante ello, la PP en pacientes con respiración espontánea no intubados podría considerarse como una estrategia ante el riesgo de que la pandemia desborde los servicios de salud, especialmente en entornos con recursos limitados. Sin embargo, al implementarse se debe garantizar un adecuado seguimiento, y una estricta monitorización clínica, para demostrar una mejoría de oxigenación y control de los signos de dificultad o esfuerzo inspiratorio marcado, evitar la lesión pulmonar autoinducida, el retardo en la intubación y el posible riesgo en incrementar la mortalidad. Por todo lo anterior se requiere mayor evidencia para demostrar en qué tipo de pacientes puede ser beneficiosa.

El uso de la CAF, con o sin PP, cuenta con bastante experiencia en pacientes con COVID-19 y falla respiratoria hipoxémica (22). Además, se ha implementado el índice ROX como indicador sencillo y disponible para la toma de decisiones en pacientes con CAF. Así, un índice ROX elevado (generalmente por encima de 4,88 a las 2, 6 y 12 horas) se asocia con el éxito de la CAF, mientras que un índice ROX reducido $(<2,85$ a las 2 horas; $<3,47$ a las 6 horas; o $<3,85$ a las 12 horas) se asociaría con fracaso, e indicaría la necesidad de intubación inmediata (22-24).

$$
\text { Índice } \mathrm{ROX}=\left(\mathrm{SpO}_{2} / \mathrm{FiO}_{2}\right) / \mathrm{FR}
$$

La alta mortalidad asociada con la ventilación mecánica invasiva descrita inicialmente, hizo considerar a muchos el uso de las anteriores estrategias no invasivas para evitar la intubación (25). Gran discusión surgió entre dos grupos con tendencias totalmente diferentes, por un lado, quienes pretendían evitar la intubación y la posible lesión inducida por el ventilador; y por el otro, quienes proponían la intubación temprana para evitar la posible lesión pulmonar autoinducida, asociada con un esfuerzo inspiratorio marcado $(26,27)$.

\section{Intubación}

En medio de la pandemia está claro que el impacto que tiene la ventilación mecánica invasiva en reducir la mortalidad es mucho mayor en pacientes jóvenes, y que esto se disminuye notoriamente con la edad y las comorbilidades del paciente. Sin embargo, la decisión de intubación debe basarse en una juiciosa evaluación de riesgo/beneficio, y la falla respiratoria en COVID-19 puede manejarse igual que la ocasionada por otras etiologías $(25,28)$.

La experiencia inicial en China sugirió criterios clínicos de intubación validados antes de la pandemia, como frecuencia respiratoria $>30 \mathrm{rpm}$; disociación toracoabdominal; hipoxemia $\mathrm{PaO}_{2} / \mathrm{FiO}_{2} \leq 150 \mathrm{~mm} \mathrm{Hg}$; no mejoría a pesar de manejo; $>2$ horas de VMNI y CAF, si la condición ha empeorado o si se prevé que empeore $(29,30)$.

A continuación, se muestra un resumen de las recomendaciones de la mayoría de guías sobre el momento de la intubación $(1,3,13,29-32)$ :

- Contar con adecuado EPP, como mascarilla N95, protección ocular, overol, doble guante largo, careta.

- Lista de chequeo diligenciada.

- Insumos, como bandeja, guía metálica, varios tubos, succión, capnografía.

- Evaluar volemia; considerar un bolo de $4 \mathrm{~mL} / \mathrm{kg}$.

- Preoxigenar con dispositivo el filtro y la máscara sin ventilar. 
Medicamentos:

- Paciente estable: fentanilo $(1,5 \mu \mathrm{g} / \mathrm{kg})$ más lidocaína $(1,5 \mathrm{mg} / \mathrm{kg})$ más propofol $(1-2 \mathrm{mg} / \mathrm{kg})$ más rocuronio $(1,2 \mathrm{mg} / \mathrm{kg})$ o succinilcolina $(1-1,5 \mathrm{mg} / \mathrm{kg})$.

- Paciente inestable: ketamina $(1,5 \mathrm{mg} / \mathrm{kg})$ más fentanilo $(1,5 \mu \mathrm{g} / \mathrm{kg})$ más rocuronio $(1,2 \mathrm{mg} / \mathrm{kg})$ o succinilcolina $(1,5 \mathrm{mg} / \mathrm{kg})$.

- Noradrenalina $(0,04 \mu \mathrm{g} / \mathrm{kg} / \mathrm{min})$, considerar temprano.

- Tener lista atropina.

Lista de chequeo $(1,3,13,29-32)$ :

- Evaluación

- ¿Vía aérea difícil?

- Compromiso fisiológico: presión arterial, oxigenación, $\mathrm{pH}$, alergias, comorbilidades.

- Asegurar insumos, equipo, plan.

- Paciente: posición, altura, dentadura, preoxigenación, hemodinamia, aspiración.

- Insumos

- Monitor: $\mathrm{SpO}_{2}, \mathrm{PA}$, electrocardiograma (ECG), capnografía.

- Equipos: bolsa con máscara, cánula de Mayo, laringoscopio, tubos, guía, lubricante, fijador de tubo, ventilador.

- Medicamentos.

- Recurso humano: líder, operador, enfermera, terapista, auxiliar.

- Plan de acción: plan A, B, C, D; medicamentos (orden, dosis, tiempos).

Sobre el proceso de intubación y posintubación algunas recomendaciones de guías son (13):

- En los médicos expertos en su uso se recomienda el videolaringoscopio (VL) rutinario para el primer intento de intubación.
- Además de que el VL puede contribuir al éxito del primer paso, visualice la laringe usando la vista indirecta (pantalla de video), con el operador de pie y el codo recto, maximiza la distancia entre la cara del operador de la vía aérea y el paciente. Esto debería reducir el riesgo de transmisión viral.

- Se debe tener cuidado al colocar el tubo a la profundidad correcta la primera vez, para minimizar la necesidad de desinflar el brazalete posterior.

- Una vez que se coloca el tubo se debe inflar el manguito antes de intentar la ventilación con presión positiva. El filtro viral debe aplicarse directamente al tubo traqueal.

- La presión del manguito debe controlarse con un manómetro de manguito para garantizar un sellado adecuado.

\section{Parámetros ventilatorios iniciales $(1,3,13,33)$}

- $\mathrm{FiO}_{2}$ : ajustar para lograr las metas de $\mathrm{SaO}_{2}$ anteriormente mencionadas, y evitar la toxicidad por oxígeno.

- Volumen corriente: se recomienda utilizar el peso predicho para establecer el volumen corriente inicial y emplear volúmenes corrientes bajos $(6 \mathrm{~mL} / \mathrm{kg})$, teniendo como metas una presión meseta $<30 \mathrm{~cm}$ de $\mathrm{H}_{2} \mathrm{O}$ y una presión de conducción $<15$.

- Presión positiva al final de la espiración (PEEP): en pacientes con síndrome de dificultad respiratoria aguda (SDRA) leve y moderado, PEEP de 5 y $10 \mathrm{~mm}$ de $\mathrm{H}_{2} \mathrm{O}$, y en pacientes con SDRA severo considerar PEEP entre 10 y $15 \mathrm{~mm} \mathrm{Hg}$ con ulterior valoración con la tabla de $\mathrm{FiO}_{2} / \mathrm{PEEP}$, la presión de conducción, la presión meseta y la distensibilidad.

\section{Sedación, analgesia, relajación (34-40)}

Luego de intubar al paciente se debe ajustar la sedación, la analgesia, la prevención y el manejo del delirio, así como considerar la relajación neuromuscular. Antes de la pandemia las guías se habían inclinado a proponer la estrategia $\mathrm{ABCDEF}$ :

a. Analgesia: monitorización, prevención y manejo del dolor.

Revista Colombiana de Neumología Vol. 32 N. ${ }^{\circ} 1$ | 2020 
b. Basar el protocolo en prueba diaria de despertar y prueba de respiración espontánea.

c. Considerar medicamentos.

d. Delirio: monitorización, prevención y manejo del delirio.

e. Ejercicio y movilidad tempranos.

f. Familia involucrada en la atención centrada en el paciente.

En general, este manejo iba encaminado a promover la analgesia sobre la sedación, a garantizar la sedación superficial o sedación consciente, y a evitar el uso de benzodiacepinas. Sin embargo, a nivel mundial, la ventilación mecánica en pacientes con COVID-19 ha planteado retos tan dramáticos como el distanciamiento paciente-familia, el riesgo de contagio para el personal de salud, el desconocimiento de una nueva enfermedad, que ha obligado a sedación profunda, uso de analgésicos, empleo de benzodiacepinas en dosis altas, y a prolongar la ventilación mecánica. Todo esto ha traído el desabastecimiento mundial de medicamentos para sedación y analgesia, lo que ha obligado al uso de medicamentos que habían sido abandonados o a nuevos medicamentos. Por ello, la AMCI y la Sociedad Colombiana de Anestesiología y Reanimación (SCARE), junto con otras sociedades científicas, han publicado un documento que propone estrategias de analgesia, sedación y relajación ante el desabastecimiento, como:

- En la fase posintubación o de sedación profunda se requiere garantizar acople del paciente/ventilador, permitir el uso de parámetros elevados e implementar estrategias, como la posición prona. Como analgésicos se han propuesto los opioides (fentanilo, remifentanilo, morfina), fenciclidinas (ketamina), no opioides, no antiinflamatorios no esteroideos (AINE) (nefopam). Como sedantes se han planteado los endovenosos (propofol, midazolam, tiopental, fenobarbital, lormetazepam) y los agentes inhalados (isoflurano, sevoflurano, desflurano). En esta fase se recomienda la relajación neuromuscular, idealmente con cisatracurio. Sin embargo, ante el desabastecimiento se han propuesto otras opciones como rocuronio, vecuronio, pancuronio o atracurio.
Se ha planteado emplearlos de manera alternante y en las dosis más bajas posibles para lograr las metas de sedación deseadas, evitar los efectos colaterales y disminuir el riesgo de desabastecimiento.

- Fase de transición: se ha logrado estabilidad hemodinámica y ventilatoria, lo que permite reducir los soportes. Se disminuyen la dosis y los medicamentos para lograr una escala RASS de 0. Para ello se han propuesto agonistas $\alpha_{2}$ (dexmedetomidina, clonidina), benzodiacepinas (lorazepam), moduladores del afecto (ácido valproico), barbitúricos (fenobarbital) y reguladores del sueño (melatonina); analgésicos opioides (hidromorfona, metadona, remifentanilo, morfina, oxicodona) y fenciclidinas (ketamina).

- Fase de sedación consciente: está claro que deberíamos volver a las medidas o estrategias que antes de la pandemia demostraron impactar en desenlaces clínicos. Tales medidas se resumen en las guías y paquetes de medidas que permiten optimizar la interacción paciente/ventilador, facilitar el retiro del ventilador y reducir las secuelas ulteriores. Así, diariamente debería evaluarse la posibilidad de retiro de sedación, y luego considerar la prueba de respiración espontánea, para definir si es posible o no el retiro de ventilador.

\section{Posición prona}

A partir del estudio PROSEVA se logró demostrar que cuando la PP se aplica en las primeras horas de un SDRA, con PaFi $<150$ reduce la mortalidad, especialmente cuando se usa con ventilación protectora, y con una duración de 16 horas o más. La literatura ulterior ha confirmado lo anterior, y hace de la PP una estrategia de calidad y obligatoria en el paciente crítico con SDRA (41-44).

\section{Traqueostomía}

Ante el temor por el contagio y la dispersión de aerosoles que genera el procedimiento, a comienzos de la pandemia se cuestionó el tiempo para realizar la traqueostomía. Sin embargo, contando con los EPP adecuados, y con las razonables medidas de precaución, se ha planteado una ventana de oportunidad para realizar la traqueostomía entre los días 10 y 21 de intubación $(45,46)$ (Tabla 2). 
Tabla 2. Intervenciones que reducen la mortalidad en los pacientes ventilados

\begin{tabular}{|c|c|c|c|}
\hline Estudio & Pacientes & Intervención & Beneficio \\
\hline ARMA (47) & 861 & Ventilación protectora & $\begin{array}{l}\text { Reducción de la mortalidad de } 40 \% \text { a } 31 \% \\
(p=0,007) ; \text { días libres de ventilación mecánica de } \\
12 \text { a } 10(p=0,007) ; \text { y días libres de falla orgánica } \\
\text { de } 15 \text { a } 12(p=0,006)\end{array}$ \\
\hline $\begin{array}{l}\text { Protocolo de } \\
\text { sedación (48) }\end{array}$ & 128 & Protocolo de despertar diario & $\begin{array}{l}\text { La duración media del ventilador fue de } 4,9 \text { días } \\
\text { frente a } 7,3(P=0,004) \text {; estancia hospitalaria de } \\
6,4 \text { días frente a 9,9 días }(P=0,02)\end{array}$ \\
\hline ACURASYS (49) & 340 & $\begin{array}{l}\text { Relajación neuromuscular en } \\
\text { SDRA }\end{array}$ & $\begin{array}{l}\text { La mortalidad a } 28 \text { días fue de } 23,7 \% \text { (IC } 95 \% \\
18,1-30,5) \text { frente a } 33,3 \% \text { (IC } 95 \% 26,5-40,9) \text { con } \\
\text { placebo }(P=0,05)\end{array}$ \\
\hline PROSEVA (50) & 466 & Ventilación prona en SDRA & $\begin{array}{l}\text { Mortalidad a } 90 \text { días de } 23,6 \% \text { frente a } 41 \% \\
(P<0,001) \text {, con un HR de } 0,44 \text { (IC } 95 \% 0,29-0,67)\end{array}$ \\
\hline $\begin{array}{l}\text { Prueba de } \\
\text { respiración } \\
\text { espontánea (51) }\end{array}$ & 300 & $\begin{array}{l}\text { Prueba de respiración } \\
\text { espontánea }\end{array}$ & $\begin{array}{l}\text { Tiempo de ventilación mecánica de } 4,5 \text { días } \\
\text { frente a } 6 \text { días }(P=0,003) \text {; tiempo de retiro del } \\
\text { ventilador de } 1 \text { día frente a } 3 \text { días }(P<0,001) \text {; } \\
\text { complicaciones en } 20 \% \text { frente a } 41 \%(P=0,001) \text {; } \\
\text { el costo total fue menor ( } \$ 15740 \text { frente a } \$ 20 \\
890 ; P=0,03)\end{array}$ \\
\hline $\mathrm{ABC}(34)$ & 336 & $\begin{array}{l}\text { Protocolo de prueba de } \\
\text { despertar y prueba de } \\
\text { respiración espontánea }\end{array}$ & $\begin{array}{l}\text { Más días sin ventilador ( } 3,1 \text { días; IC } 95 \% 0,7-5,6 \text {; } \\
p=0,02 \text { ) y se dieron de alta de UCI antes }(9,1 \\
\text { días frente a } 12,9 \text { días; } p=0,01) ; \text { y del hospital } \\
\text { antes }(14,9 \text { días frente a } 19,2 \text { días; } p=0,04) \text {. } \\
\text { Menos probabilidades de morir (HR 0,68; IC } 95 \% \\
0,50-0,92 ; p=0,01) \text {. Número de casos necesario } \\
\text { a tratar }(\mathrm{NNT})=7,4 ; \text { IC } 95 \% 4,2-35,5\end{array}$ \\
\hline $\begin{array}{l}\text { RECOVERY Uso de } \\
\text { dexametasona (11) }\end{array}$ & 2104 & $\begin{array}{l}\text { Tratamiento habitual frente a } \\
\text { tratamiento habitual más } 6 \mathrm{mg} \\
\text { de dexametasona } 1 \text { vez al día } \\
\text { (oral o intravenosa) durante un } \\
\text { máximo de } 10 \text { días (o hasta el } \\
\text { alta, si es antes) }\end{array}$ & $\begin{array}{l}454(21,6 \%) \text { pacientes asignados a la intervención } \\
\text { con dexametasona y } 1065(24,6 \%) \text { pacientes } \\
\text { asignados al tratamiento habitual murieron a los } \\
28 \text { días (índice de frecuencia ajustada por edad, } \\
\text { RR 0,83; IC 95\% 0,74-0,92; } P<0,001 \text { ) }\end{array}$ \\
\hline
\end{tabular}

\section{Criterios de egreso de $\mathrm{UCI}$}

Tolerancia a la extubación al menos por 48-72 horas; disminución del aporte de oxígeno con saturación de oxígeno $>90 \%$ por más de 48 horas; sin requerimientos de soporte inotrópico o vasopresores; tolerancia de la vía oral; ausencia de fiebre $>72$ horas sin antipiréticos $(1,3,13)$.

\section{Referencias}

1. Accini JL, Beltrán N, Nieto VH, Ramos E, Pizarro C, Rebolledo $\mathrm{CE}$, et al. Declaración de consenso en medicina crítica para la atención multidisciplinaria del paciente con sospecha o confirmación diagnóstica de COVID-19. Acta Colomb Cuid Intensivo. 2020.20;4:287-333. doi: 10.1016/j. acci.2020.04.003.

2. Jibaja M, Ortiz-Ruiz G, García F, Garay-Fernández M, Montelongo F, Martínez J, et al. Hospital mortality and effect of adjusting $\mathrm{PaO} 2 / \mathrm{FiO} 2$ according to altitude above the sea level in acclimatized patients undergoing invasive mechanical ventilation. A multicenter study. Arch Bronconeumol. 2020;56(4):218-24. doi: 10.1016/j.arbres.2019.06.024.

3. Saavedra $\mathrm{CH}$. Consenso colombiano de atención, diagnóstico y manejo de la infección por SARS-CoV-2/COVID-19 en establecimientos de atención de la salud. Recomendaciones basadas en consenso de expertos e informadas en la evidencia. Rev Asoc Colomb Infectol. 2020;24(3). doi: 10.22354/ in.v24i3.851. 
4. Siemieniuk RAC, Chu DK, Kim LHY, Güell-Rous MR, Alhazzani W, Soccal PM, et al. Oxygen therapy for acutely ill medical patients: a clinical practice guideline. BMJ. 2018;363:k4169. doi: 10.1136/bmj.k4169.

5. Alhazzani W, Møller MH, Arabi YM, Loeb M, Gong MN, Fan E, et al. Surviving sepsis campaign: guidelines on the management of critically ill adults with Coronavirus Disease 2019 (COVID-19). Intensive Care Med. 2020;46:854-87. doi: 10.1007/s00134-020-06022-5.

6. Bhimraj A, Morgan RL, Shumaker AH, Lavergne V, Baden L, Cheng VC, et al. Infectious diseases Society of America Guidelines on the treatment and management of patients with COVID-19. Clin Infect Dis. 2020:ciaa478. doi: 10.1093/cid/ ciaa478.

7. Fragkou PC, Belhadi D, Peiffer-Smadja N, Moschopoulos CD, Lescure FX, Janocha H, Karofylakis E, Yazdanpanah Y, Mentré F, Skevaki C, Laouénan C, Tsiodras S; ESCMID Study Group for Respiratory Viruses. Review of trials currently testing treatment and prevention of COVID-19. Clin Microbiol Infect. 2020 Aug;26(8):988-98. doi: 10.1016/j. cmi.2020.05.019.

8. Murthy S, Gomersall CD, Fowler RA. Care for critically ill patients with COVID-19. JAMA. 2020;323(15):1499-500. doi: 10.1001/jama.2020.3633.

9. Siemieniuk RA, Bartoszko JJ, Ge L, Zeraatkar D, Izcovich A, Kum E, et al. Drug treatments for covid-19: living systematic review and network meta-analysis. BMJ. 2020;370:m2980. doi: 10.1136/bmj.m2980.

10. WHO Solidarity Trial Consortium, Pan H, Peto R, HenaoRestrepo AM, Preziosi MP, Sathiyamoorthy V, et al. Repurposed antiviral drugs for Covid-19 - Interim WHO Solidarity Trial Results. N Engl J Med. 2020:NEJMoa2023184. doi: 10.1056/NEJMoa2023184.

11. RECOVERY Collaborative Group, Horby P, Lim WS, Emberson JR, Mafham M, Bell JL, et al. Dexamethasone in hospitalized patients with Covid-19 - Preliminary report. N Engl J Med. 2020:NEJMoa2021436. doi: 10.1056/NEJMoa2021436.

12. Ji Y, Ma Z, Peppelenboshch M, Pan Q. Potential association between COVID-19 mortatlity and health-care resource availability. Lancet Glob Health. 2020;04;8:e48. doi: 10.1016/ S2214-109X(20)30068-1.

13. Ministerio de Salud y Protección Social. Lineamientos para el manejo clínico de pacientes con infección por nuevo coronavirus COVID 19. 2020. Disponible en: https://www.minsalud. gov.co/Ministerio/Institucional/Procesos $\% 20 \mathrm{y} \% 20$ procedimientos/PSSS03.pdf

14. Douglas WW, Rehder K, Beynen FM, Sessler AD, Marsh HM. Improved oxygenation in patients with acute respiratory failure: the prone position. Am Rev Respir Dis. 1977;115(4):55966. doi: 10.1164/arrd.1977.115.4.559.

15. Gattinoni L, Busana M, Giosa L, Macrì MM, Quintel M. Prone positioning in acute respiratory distress syndrome. Semin Respir Crit Care Med. 2019;40(1):94-100. doi: 10.1055/s0039-1685180.
16. Scaravilli V, Grasselli G, Castagna L, Zanella A, Isgrò S, Lucchini A, et al. Prone positioning improves oxygenation in spontaneously breathing nonintubated patients with hypoxemic acute respiratory failure: A retrospective study. J Crit Care. 2015;30(6):1390-4. doi: 10.1016/j.jcrc.2015.07.008.

17. Ding L, Wang L, Ma W, He H. Efficacy and safety of early prone positioning combined with HFNC or NIV in moderate to severe ARDS: a multi-center prospective cohort study. Crit Care. 2020;24:28. doi: 10.1186/s13054-020-2738-5.

18. Koeckerling D, Barker J, Mudalige NL, Oyefeso O, Pan D, Pareek M, et al. Awake prone positioning in COVID-19. Thorax. 2020;75:833-4. doi: 10.1136/thoraxjnl-2020-215133.

19. Remuzzi A, Remuzzi G. COVID-19 and Italy: what next? Lancet. 2020;395:1225-8. doi: 10.1016/S0140-6736(20)30627-9.

20. Li JL, Fink JB, Ehrmann S: High-flow nasal cannula for COVID-19 patients: low risk of bio-aerosol dispersion. Eur Respir J. 2020;55(5):2000892. doi: 10.1183/13993003.00892-2020.

21. Winck JC, Ambrosino N. COVID-19 pandemic and non-invasive respiratory management: Every Goliath needs a David. An evidence based evaluation of problems. Pulmonology. 2020;26(4):213-20. doi: 10.1016/j.pulmoe.2020.04.013.

22. Agarwal A, Basmaji J, Muttalib F, Granton D. High-flow nasal cannula for acute hypoxemic respiratory failure in patients with COVID-19: systematic reviews of effectiveness and its risks of aerosolization, dispersion, and infection transmission. Can J Anaesth. 2020;67(9):1217-48. doi: 10.1007/s12630020-01740-2.

23. Ricard JD, Roca O, Lemiale V, Corley A, Braunlich J, Jones $P$, et al. Use of nasal high flow oxygen during acute respiratory failure. Intensive Care Med. 2020;46(12):2238-47. doi: 10.1007/s00134-020-06228-7.

24. Raoof S, Nava S, Carpati C, Hill NS. High-flow, noninvasive ventilation and awake (nonintubation) proning in patients with coronavirus disease 2019 with respiratory failure. Chest. 2020;158(5):1992-2002. doi: 10.1016/j.chest.2020.07.013.

25. Wunsch H. Mechanical ventilation in COVID-19: Interpreting the current epidemiology. Am J Respir Crit Care Med. 2020;202(1):1-4. doi: 10.1164/rccm.202004-1385ED.

26. Tobin MJ, Laghi F, Jubran A. Caution about early intubation and mechanical ventilation in COVID-19. Ann Intensive Care. 2020;10(1):78. doi: 10.1186/s13613-020-00692-6.

27. Gattinoni L, Marini JJ, Busana M, Chiumello D, Camporota L. Spontaneous breathing, transpulmonary pressure and mathematical trickery. Ann Intensive Care. 2020;10:88. doi: 10.1186/s13613-020-00708-1.

28. Hernandez-Romieu AC, Adelman MW, Hockstein MA, Robichaux CJ, Edwards JA, Fazio JC, et al. Timing of intubation and mortality among critically ill coronavirus disease 2019 patients: A single-center cohort study. Crit Care Med. 2020;48(11):e1045-53. doi: 10.1097/ CCM.0000000000004600.

29. Zuo MZ, Huang YG, Ma WH, Xue ZG, Zhang JQ, Gong YH, et al. Expert recommendations for tracheal intubation in cri- 
tically ill patients with noval coronavirus disease 2019. Chin Med Sci J. 2020;35(2):105-9. doi: 10.24920/003724.

30. Meng L, Qiu H, Wan L, Ai Y, Xue Z, Guo Q, et al. Intubation and ventilation amid the COVID-19 outbreak: Wuhan's experience. Anesthesiology. 2020;132(6):1317-32. doi: 10.1097/ ALN.0000000000003296.

31. Sorbello M, El-Boghdadly K, Di Giacinto I, Cataldo R. The Italian coronavirus disease 2019 outbreak: recommendations from clinical practice. Anaesthesia. 2020;75(6):724-32. doi: 10.1111/anae.15049.

32. Cook TM, El-Boghdadly K, McGuire B, McNarry AF, Patel A, Higgs A. Consensus guidelines for managing the airway in patients with covid-19. Anaesthesia. 2020;75:785-99. doi: 10.1111/anae.15054.

33. Ortiz-Ruiz G, Dueñas-Castell C, Garay-Fernandez M, LaraGarcia A, Varón F, et al. Consenso colombiano de síndrome de dificultad respiratoria aguda (SDRA) «Documento de Rionegro 2019». Acta Colomb Cuid Intensivo. 2020;20:200-52. doi: 10.1016/j.acci.2020.03.001.

34. Ely EW. The ABCDEF bundle: science and philosophy of how ICU liberation serves patients and families. Crit Care Med. 2017;45(2):321-30. doi: 10.1097/CCM.0000000000002175.

35. Barnes-Daly MA, Phillips G, Ely EW. Improving hospital survival and reducing brain dysfunction at seven California community hospitals: implementing pad guidelines via the ABCDEF bundle in 6,064 patients. Crit Care Med. 2017;45:171-8. doi: 10.1097/CCM.0000000000002149.

36. Pun BT, Balas MC, Barnes-Daly MA, Thompson JL, et als: Caring for critically ill patients with the ABCDEF bundle: results of the ICU liberation collaborative in over 15,000 adults. Crit Care Med.2019;47:3-14. doi: 10.1097/ CCM.0000000000003482.

37. Devlin JW, Skrobik Y, Gelinas C, Needham DM, et als: Clinical practice guidelines for the prevention and management of pain, agitation/sedation, delirium, immobility, and sleep disruption in adult patients in the ICU. Crit Care Med. 2018;46:e825-73. doi: 10.1097/CCM.0000000000003299.

38. Celis-Rodríguez E, Díaz JC, Cárdenas YR, Carrizosa JA, Pinilla DI, Ferrer LE, et al. Guías de práctica clínica basadas en la evidencia para el manejo de la sedoanalgesia y delirium en el paciente adulto críticamente enfermo. Med Intensiva. 2020;44:171-84. doi: 10.1016/j.medin.2019.07.013.

39. Papazian L, Forel JM, Gacouin A, Penot-Ragon C, Perrin G, Loundou A, et al. Neuromuscular blockers in early acute respiratory distress syndrome. N Engl J Med. 2010;363(12):110716. doi: 10.1056/NEJMoa1005372.

40. Alhazzani W, Belley-Cote E, Moller MH, Angus DC, Papazian L, Arabi YM, et al. Neuromuscular blockade in patients with ARDS: a rapid practice guideline. Intensive Care Med. 2020;46:1977-86. doi: 10.1007/s00134-020-06227-8.

41. Munshi L, Del Sorbo L, Adhikari NKJ, Hodgson CL, Wunsch $\mathrm{H}$, Meade $\mathrm{MO}$, et al. Prone position for acute respiratory distress syndrome. A systematic review and meta-analysis. Ann Am Thorac Soc. 2017;14(4):S280-8. doi: 10.1513/ AnnalsATS.201704-343OT.

42. Guerin C, Reignier J, Richard JC, Beuret P, Gacouin A, Boulain $\mathrm{T}$, et al. Prone positioning in severe acute respiratory distress syndrome. N Engl J Med. 2013;368(23):2159-68. doi: 10.1056/NEJMoa1214103.

43. Sud S, Friedrich JO, Adhikari NK, Taccone P, Mancebo J, Polli F, et al. Effect of prone positioning during mechanical ventilation on mortality among patients with acute respiratory distress syndrome: a systematic review and meta-analysis. Can Med Assoc J. 2014;186(10):E381-90. doi: 10.1503/ cmaj. 140081.

44. Bloomfield R, Noble DW, Sudlow A. Prone position for acute respiratory failure in adults. Cochrane Database Syst Rev. 2015;2015(11):CD008095. doi: 10.1002/14651858. CD008095.pub2.

45. Lamb CR, Desai NR, Angel L, Chaddha U, Sachdeva A, Sethi $\mathrm{S}$, et al. Use of tracheostomy during the COVID-19 pandemic: CHEST/AABIP/AIPPD: Expert Panel Report. CHEST. 2020;158(4):1499-514. doi: 10.1016/j.chest.2020.05.571.

46. McGrath BA, Brenner MJ, Warrillow SJ, Pandian V, Arora A, Cameron TS, et al. Tracheostomy in the COVID-19 era: global and multidisciplinary guidance. Lancet Respir Med. 2020;8(7):717-25. doi: 10.1016/S2213-2600(20)30230-7.

47. Singer BD, Jain M, Budinger GRX, Wunderink RG. A call for rational intensive care in the era of COVID-19. AJRCMB. 2020;63(1). doi: 10.1165/rcmb.2020-0151LE.

48. Warwicker P. Polio: the story of the great polio epidemic in Copenhagen in 1952. Copenhagen, Denmark: Gyldendal; 2006.

49. Gensowski M, Nielsen TH, Nielsen NM, Rosin-Slater M, Wüstk M. Childhood health shocks, comparative advantage, and long-term outcomes: evidence from the last Danish polio epidemic. J Health Econom. 2019;66:27-36. doi: 10.1016/j. jhealeco.2019.03.010.

50. Bellani G, Laffey JG, Pham T, Madotto F, Fan E, Brochard L, et al. Noninvasive ventilation of patients with acute respiratory distress syndrome: insights from the LUNG SAFE study. Am J Respir Crit Care Med. 2017;195:67-77. doi: 10.1164/ rccm.201606-1306OC.

51. Mehta AB, Douglas IS, Walkey AJ. Evidence-based utilization of noninvasive ventilation and patient outcomes. Ann Am Thorac Soc. 2017;14:1667-73. doi: 10.1513/ AnnalsATS.201703-208OC. 